\title{
The $[\mathrm{Zn} / \mathrm{Fe}]-[\mathrm{Fe} / \mathrm{H}]$ trend for disk and halo stars ${ }^{\star}$
}

\author{
Y. Q. Chen ${ }^{1}$, P. E. Nissen ${ }^{2}$, and G. Zhao ${ }^{1}$ \\ 1 National Astronomical Observatories, Chinese Academy of Sciences, Beijing 100012, PR China \\ e-mail: [cyq;zg]@bao.ac.cn \\ 2 Institute of Physics and Astronomy, University of Aarhus, 8000 Aarhus C, Denmark \\ e-mail: pen@phys.au.dk
}

Received 4 February 2004 / Accepted 17 June 2004

\begin{abstract}
Zn abundances, derived from a model atmosphere analysis of the $\lambda 6362.35 \AA \mathrm{Zn}$ I line, are presented for 44 thin disk, 10 thick disk and 8 halo dwarf stars in the metallicity range $-1.0<[\mathrm{Fe} / \mathrm{H}]<+0.2$. It is found that $[\mathrm{Zn} / \mathrm{Fe}]$ in thin disk stars shows a slight increasing trend with decreasing metallicity reaching a value $[\mathrm{Zn} / \mathrm{Fe}] \simeq+0.1$ at $[\mathrm{Fe} / \mathrm{H}]=-0.6$. The thick disk stars in the metallicity range $-0.9<[\mathrm{Fe} / \mathrm{H}]<-0.6$ have an average $[\mathrm{Zn} / \mathrm{Fe}] \simeq+0.15 \mathrm{dex}$, whereas five alpha-poor and Ni-poor halo stars in the same metallicity range have $[\mathrm{Zn} / \mathrm{Fe}] \simeq 0.0$ dex. These results indicate that $\mathrm{Zn}$ is not an exact tracer of $\mathrm{Fe}$ as often assumed in abundance studies of damped Lyman-alpha systems (DLAs). A better understanding of the nucleosynthesis of $\mathrm{Zn}$ is needed in order to obtain more detailed information on the past history of star formation in DLAs from e.g. the observed sulphur/zinc ratio.
\end{abstract}

Key words. stars: abundances - stars: atmospheres - Galaxy: evolution - Galaxy: solar neighbourhood - galaxies: high-redshift

\section{Introduction}

The determination of zinc abundances in Galactic stars is of high interest in astrophysics for at least two reasons. Firstly, the nucleosynthesis of zinc is not well understood. According to Woosley \& Weaver (1995) Zn is produced by supernovae ( $\mathrm{SNe}$ ) of type II from two processes: i) neutron capture on iron group nuclei during $\mathrm{He}$ and $\mathrm{C}$-burning (the weak $s$-process; Langer et al. 1989); and ii) alpha-rich freeze-out following nuclear statistical equilibrium in layers heated to more than $5 \times 10^{9} \mathrm{~K}$. However, as shown by Timmes et al. (1995), and Goswami \& Prantzos (2000), the corresponding yields underpredicts $\mathrm{Zn} / \mathrm{Fe}$ by about a factor of two compared to the observed ratio in Galactic halo stars. Furthermore, the yield corresponding to the first process is metallicity dependent leading to a predicted rise of $[\mathrm{Zn} / \mathrm{Fe}]$ as a function of $[\mathrm{Fe} / \mathrm{H}]$ for disk stars in disagreement with the rather flat trend of $[\mathrm{Zn} / \mathrm{Fe}]$ vs. $[\mathrm{Fe} / \mathrm{H}]$ observed by Sneden et al. (1991). In order to get a better agreement with the observed trend, Matteucci et al. (1993) argued that type Ia $\mathrm{SNe}$ give a very significant contribution to the production of Zn, but according to Iwamoto et al. (1999) standard models of type Ia SNe produce little $\mathrm{Zn}$. As an alternative source of Zn, Hoffman et al. (1996) suggested that a significant amount of zinc (together with light $p$-process nuclei) could be produced in the neutrino-powered wind of 10 to 20 solar mass $\mathrm{SNe}$, essentially the same site as proposed

* Based on observations collected at the National Astronomical Observatories, Xinglong, China and the European Southern Observatory, La Silla, Chile (ESO No. 67.D-0106). for the $r$-process (Woosley et al. 1994) but at earlier times after the explosion. Furthermore, Umeda \& Nomoto (2002) have discussed how the produced $\mathrm{Zn} / \mathrm{Fe}$ ratio in massive metal-poor $\mathrm{SNe}$ depends on the mass cut, neutron excess and explosion energy, in an attempt to explain the high $[\mathrm{Zn} / \mathrm{Fe}]$ values observed for the most metal-poor stars (Primas et al. 2000; Cayrel et al. 2004).

Secondly, zinc is a key element in studies of abundances in damped Ly $\alpha$ (DLA) systems, because it is the only element in the iron-peak group, which is undepleted onto dust in the interstellar medium. With reference to the data of Sneden et al. (1991), Zn is often taken as a proxy for Fe in DLA studies to derive dust depletion factors (e.g., Vladilo 2002) and to date the the star formation process at high $z$ from $[\alpha / \mathrm{Fe}]$, i.e. the logarithmic ratio between the abundance of alpha-elements $(\mathrm{O}, \mathrm{Ne}$, $\mathrm{Mg}, \mathrm{Si}, \mathrm{S}, \mathrm{Ca}, \mathrm{Ti}$ ) and the abundance of iron (e.g., Pettini et al. 1999; Centurión et al. 2000). Given the uncertainty about the nucleosynthetic origin of zinc doubts have, however, been raised about the reliability of this method (Prochaska 2003; Fenner et al. 2004).

The often cited conclusion by Sneden et al. (1991) that zinc abundances closely track the overall metallicity with no discernible change in $[\mathrm{Zn} / \mathrm{Fe}]$ in the range $-2.9<[\mathrm{Fe} / \mathrm{H}]<-0.1$ has been challenged in a number of recent studies. Prochaska et al. (2000) found a mild enhancement of $\mathrm{Zn}$ relative to $\mathrm{Fe}([\mathrm{Zn} / \mathrm{Fe}] \sim+0.1)$ in ten thick disk stars with metallicities between -1.2 and -0.4 . Mishenina et al. (2002) have published a survey of $\mathrm{Zn}$ abundances in 90 disk and halo stars, and although they conclude that the data "confirms the well-known fact that 
$[\mathrm{Zn} / \mathrm{Fe}]$ is almost solar at all metallicities", Nissen (2004) notes that there is a tendency for thick disk stars in the metallicity range $-1.0<[\mathrm{Fe} / \mathrm{H}]<-0.5$ to be overabundant in $\mathrm{Zn}$. In addition, Reddy et al. (2003) have found a slightly increasing $[\mathrm{Zn} / \mathrm{Fe}$ ] with decreasing metallicity for 181 thin disk stars in the metallicity range $-0.8<[\mathrm{Fe} / \mathrm{H}]<+0.1$, and Bensby et al. (2003) found evidence of a separation of $[\mathrm{Zn} / \mathrm{Fe}]$ between thin and thick disk stars as well as a tendency of an uprising $[\mathrm{Zn} / \mathrm{Fe}]$ at $[\mathrm{Fe} / \mathrm{H}]>0$. Among halo stars, Nissen et al. (2004) derived $[\mathrm{Zn} / \mathrm{Fe}] \sim+0.1$ for stars in the metallicity range $-2.5<[\mathrm{Fe} / \mathrm{H}]<-2.0$, and as noted above Primas et al. (2000) and Cayrel et al. (2004) have found clear evidence of increasing $[\mathrm{Zn} / \mathrm{Fe}]$ values below $[\mathrm{Fe} / \mathrm{H}] \simeq-2.5$ with $[\mathrm{Zn} / \mathrm{Fe}]$ reaching a value of +0.5 dex at $[\mathrm{Fe} / \mathrm{H}] \simeq-4.0$.

In most of these works, the zinc abundances are primarily based on the $\lambda \lambda 4722.16,4810.54 \AA \mathrm{Zn}_{\mathrm{I}}$ lines, although the weak $\mathrm{Zn}$ I line at $6362.35 \AA$ is included in some of the disk star studies. A problem with the $\lambda \lambda 4722.16,4810.54 \AA \mathrm{Zn}_{\mathrm{I}}$ lines is that they are rather strong at solar metallicities $(E W \sim$ $70-80 \mathrm{~m} \AA$ in the solar flux spectrum) and blended by several weak lines in the wings. This makes it difficult to set a reliable continuum and to measure their equivalent widths accurately. The derived $\mathrm{Zn}$ abundances depend furthermore critically on the assumed value of the Van der Waals damping constant. This means that the trend of $[\mathrm{Zn} / \mathrm{Fe}]$ versus $[\mathrm{Fe} / \mathrm{H}]$ for disk stars becomes rather uncertain if the $\lambda \lambda 4722.16,4810.54 \AA \mathrm{Zn}$ I lines are included in the analysis.

In the present paper we derive zinc abundances exclusively from the weak $6362.35 \AA \mathrm{Zn}$ I line, for which the equivalent width ranges from a few $\mathrm{m} \AA$ in the most metal-poor disk stars to about $30 \mathrm{~m} \AA$ in the metal-rich stars. Hence, the line is rather ideal for accurate abundance determinations being insensitive to microturbulence and Van der Waals damping parameters. In the following Sect. 2, high resolution observations of this line is presented. Section 3 describes the model atmosphere analysis of the data and possible errors are discussed in Sect. 4. The results are discussed in Sect. 5.

\section{Observations and data reduction}

Two sets of stars are included in this paper. The first set consists mainly of thin disk stars from Chen et al. (2000, 2002), who determined abundances of solar-type dwarfs selected from the $u v b y-\beta$ photometric catalogues of Olsen $(1983,1993,1994)$. The stars have $5600 \leq T_{\text {eff }} \leq 6400 \mathrm{~K}, \log g \geq 3.8$ and $-1.0 \leq[\mathrm{Fe} / \mathrm{H}] \leq+0.3$. In Chen et al. (2000) chemical abundances of $\mathrm{O}, \mathrm{Na}, \mathrm{Mg}, \mathrm{Al}, \mathrm{Si}, \mathrm{K}, \mathrm{Ca}, \mathrm{Ti}, \mathrm{V}, \mathrm{Cr}, \mathrm{Fe}, \mathrm{Ni}$ and $\mathrm{Ba}$ were derived from high-resolution spectra of 90 disk stars. Thirty of these spectra are of sufficiently high quality to allow measurements of the equivalent width of the $6362.35 \AA$ $\mathrm{Zn}$ I line with good accuracy. In Chen et al. (2002) S, Si and $\mathrm{Fe}$ abundances were derived for 26 disk stars; 14 of these are included in the present paper. As described in the above papers, the spectra were obtained with the Coudé Echelle Spectrograph attached to the $2.16 \mathrm{~m}$ telescope at the National Astronomical Observatories (Xinglong, China). The resolution is 37000 and the signal-to-noise ratio is generally over 200 in the region of the $6362.35 \AA \mathrm{Zn}$ I line.

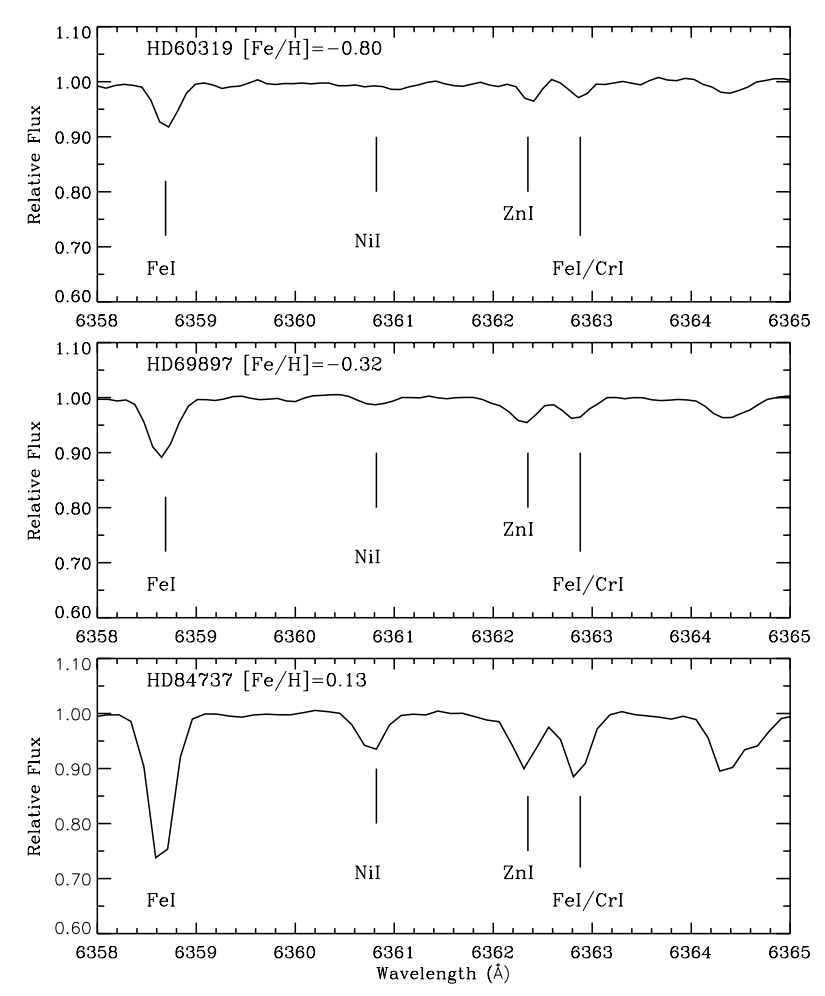

Fig. 1. A sequence of spectra around the $\lambda 6362.35 \AA \mathrm{Zn}$ I line observed with the Coudé Echelle Spectrograph attached to the $2.16 \mathrm{~m}$ telescope at the National Astronomical Observatories, Xinglong, China.

The second set of stars consists of 19 disk and halo stars with overlapping metallicities in the range $-1.0<[\mathrm{Fe} / \mathrm{H}]<-0.4$ taken from the work of Nissen \& Schuster (1997), who determined various abundance ratios from high resolution $(R=60000, S / N \sim 150-200)$ spectra obtained with the EMMI spectrograph on the ESO $3.5 \mathrm{~m}$ NTT telescope. The stars were originally selected from the catalogues of $u v b y-\beta$ photometry by Schuster \& Nissen (1988) and Schuster et al. (1993), and have atmospheric parameters in the range $5400 \leq T_{\text {eff }} \leq 6300 \mathrm{~K}$ and $4.0<\log g<4$.6. According to the value of the Galactic rotational velocity component, $V_{\text {rot }}$, the stars were classified as belonging either to the Galactic halo $\left(V_{\text {rot }}<50 \mathrm{~km} \mathrm{~s}^{-1}\right)$ or the thick disk $\left(V_{\text {rot }}>150 \mathrm{~km} \mathrm{~s}^{-1}\right)$.

For details about the reduction of the spectra we refer to the papers by Chen et al. $(2000,2002)$ and Nissen \& Schuster (1997). A special problem with the $\lambda 6362.35 \AA \mathrm{Zn}_{\text {I }}$ line is that it lays in the midst of a very broad and shallow absorption feature identified by Michell \& Mohler (1965) as due to a Ca r auto-ionization line with a central wavelength of $6361.8 \AA$. In the solar flux spectrum (Kurucz et al. 1984) the central depth of the line is about $5 \%$ and the total width is more than $15 \AA$. By fitting a polynomial function to this broad line we have rectified the spectra around the $\lambda 6362.35 \AA \mathrm{Zn}$ I line. Figure 1 shows the resulting spectra for three stars of different metallicities observed with the $2.16 \mathrm{~m}$ telescope at Xinglong, and Fig. 2 shows two representative spectra observed with the ESO NTT.

The equivalent width of the $\lambda 6362.35 \AA \mathrm{Zn}$ I line was measured in the rectified spectra by Gaussian fitting so that the contribution from the nearby $\mathrm{FeI} / \mathrm{CrI}$ line at $6362.88 \AA \mathrm{can}$ 


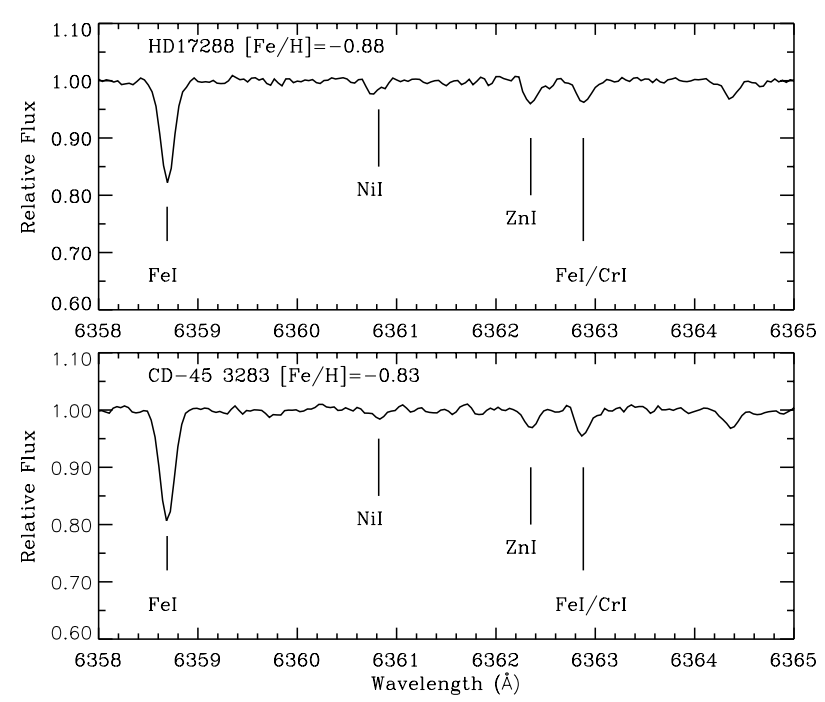

Fig. 2. Two spectra around the $\lambda 6362.35 \AA \mathrm{Zn}$ I line observed with the EMMI spectrograph on the ESO $3.5 \mathrm{~m}$ telescope, The two stars have about the same values of $T_{\text {eff }} \log g$ and $[\mathrm{Fe} / \mathrm{H}]$. Still, the $\lambda 6362.35 \AA$ $\mathrm{Zn}$ I line appears stronger in HD 17288 (a thick disk star) than in CD-45 3283 (an alpha-poor halo star).

be nearly avoided. The equivalent width for the Sun $(E W=$ $22.4 \mathrm{~m} \AA$ ), was obtained from a Moon spectrum observed at Xinglong in the same way as the programme stars. This value is consistent with the equivalent width of the $\mathrm{Zn}$ I line obtained from the the solar flux spectrum of Kurucz et al. (1984).

The measured equivalent widths are given in Tables 1 and 2 . Some of the stars are included in the ELODIE public library of high resolution spectra (Prugniel \& Soubiran 2001). With a resolution of $R=42000$ and a $S / N \sim 150-200$ these spectra are of similar quality as our spectra and as a check, equivalent widths of the $\lambda 6362.35 \AA \mathrm{Zn}$ I line have been measured in the ELODIE spectra also and are listed in Tables 1 and 2. Figure 3 shows a comparison between the two sets of equivalent widths. As seen the agreement is quite satisfactory. The rms scatter around the $1: 1$ line is $1.8 \mathrm{~m} \AA$ suggesting that the error of our equivalent measurements is around $1.3 \mathrm{~m} \AA$.

\section{Analysis}

Stellar parameters, $T_{\text {eff }}$ and $\log g$, are taken from the abovementioned papers from which the stars were selected. In Chen et al. $(2000,2002), T_{\text {eff }}$ was determined from the $b-y$ colour index using the IRFM calibration of Alonso et al. (1996) and $\log g$ was calculated from the Hipparcos parallax. In Nissen \& Schuster (1997), $T_{\text {eff }}$ was determined from the excitation balance of $\mathrm{Fe}$ I lines but it was checked that the values agree quite well with effective temperatures derived from $b-y$. The average difference, $T_{\text {exc. }}-T_{b-y}$, is $65 \mathrm{~K}$ and the rms scatter of the difference is $\pm 55 \mathrm{~K}$. As discussed later (see Table 3 ) this systematic difference in $T_{\text {eff }}$ corresponds to a change in $\left[\frac{\mathrm{Zn}}{\mathrm{Fe}}\right]$ of about 0.02 dex - quite negligible compared to other error sources. Concerning the gravity parameter, we note that some stars in Nissen \& Schuster are too distant to have accurate parallaxes. Hence, $\log g$ was determined from the ionization balance of $\mathrm{Fe}$ I and $\mathrm{Fe}$ II lines. Fourteen of the 19 stars

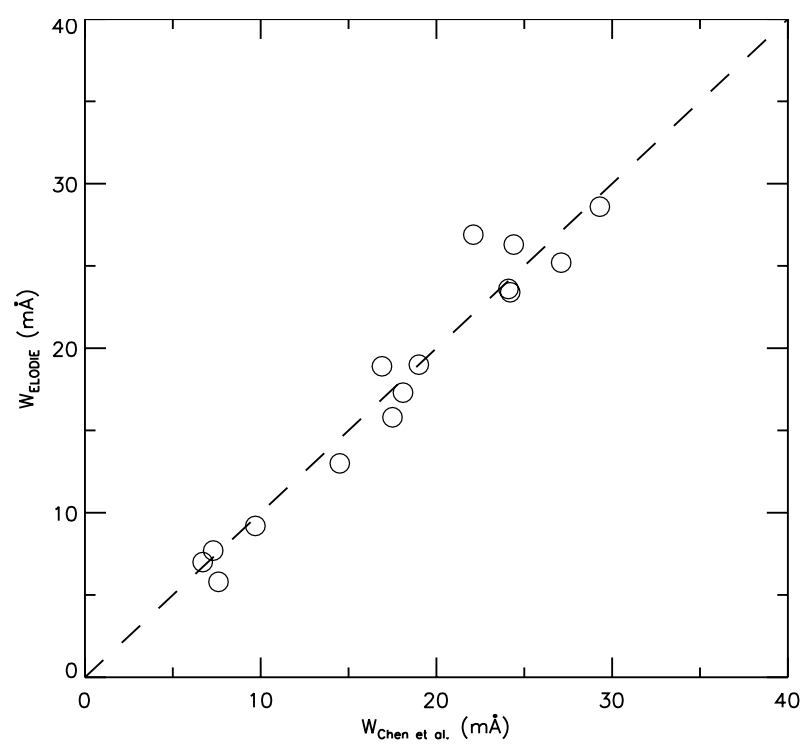

Fig. 3. The equivalent width of the $\lambda 6362.35 \AA \mathrm{Zn}_{\text {I }}$ line as measured from ELODIE spectra (Prugniel \& Soubiran 2001) versus the equivalent width from our spectra.

in Table 2 have, however, Hipparcos parallaxes with a relative error less than $25 \%$, corresponding to a $\log g$ error less than about 0.20 dex. The mean difference between the spectroscopic and parallax-derived gravity is 0.03 dex with a rms scatter of \pm 0.15 dex. The corresponding difference in $\left[\frac{\mathrm{Zn}}{\mathrm{Fe}}\right]$ is negligible. Hence, we conlcude that the different ways of deriving stellar parameters will not introduce significant effects in the abundance determination. Furthermore, we note that one star that is in common between the two samples, HD 60319 (=G088-040), has exactly the same abundance ratios in Tables 1 and 2, but this is of course a fortuitous agreement.

Our determination of abundances is based on 1D model atmospheres computed with the MARCS code using updated continuous opacities (Asplund et al. 1997) and including UV line blanketing by millions of absorption lines. LTE is assumed both in constructing the models and in deriving abundances. For all stars the microturbulence parameter $\xi_{\mathrm{t}}$ was determined by requesting that the iron abundance derived from $\mathrm{Fe}_{\mathrm{I}}$ lines should be independent of the equivalent width.

For stars selected from Chen et al. (2002) we have adopted the $[\mathrm{Fe} / \mathrm{H}]$ values given in that paper. These iron abundances are based on equivalent widths of 18 weak Fe II lines analyzed in a differential way with respect to the Sun. For stars in Chen et al. (2000), we redetermined the iron abundances using equivalent widths of a subset of 8 of the 18 Fe II lines. The abundances are nearly the same as those presented in Chen et al. (2000) based on Fe I lines. For the Nissen \& Schuster (1997) stars we use their $[\mathrm{Fe} / \mathrm{H}]$ values as determined from $104 \mathrm{Fe}_{\mathrm{I}}$ and $12 \mathrm{Fe}$ II lines (forced to give the same iron abundance due to the way the gravity was determined).

In deriving zinc abundances from the $\lambda 6362.35 \AA \mathrm{Zn}$ I line $\left(\chi_{\mathrm{exc}}=5.79 \mathrm{eV}\right)$, we have adopted $\log g f=0.14$ as determined by Biémont \& Godefroid (1980) from multi-configurational Hartree-Fock calculations. Using the MARCS model atmosphere for the Sun and an equivalent width of $22.4 \mathrm{~m} \AA$ of 
Table 1. Stellar parameters, equivalent widths of the $6362.35 \AA$ $\mathrm{Zn}$ I line and derived abundance ratios $[\mathrm{Zn} / \mathrm{Fe}]$ and $[\mathrm{Ni} / \mathrm{Fe}]$ for disk stars observed with the Xinglong $2.16 \mathrm{~m}$ telescope. $W_{a}$ is the equivalent width measured from the Xinglong spectra and $W_{b}$ the value obtained from ELODIE spectra. The stars are classified as belonging to the thin disk (D) or the thick disk population (TD) according to their kinematics.

\begin{tabular}{|c|c|c|c|c|c|c|c|c|}
\hline & & $\begin{array}{c}T_{\mathrm{eff}} \\
\mathrm{K}\end{array}$ & & & $\begin{array}{r}W_{a} \\
\mathrm{~m} \AA\end{array}$ & $\begin{array}{c}W_{b} \\
\mathrm{~m} \AA\end{array}$ & {$\left[\frac{\mathrm{Zn}}{\mathrm{Fe}}\right]$} & {$\left[\frac{\mathrm{Ni}}{\mathrm{Fe}}\right]$} \\
\hline Sun & $\mathrm{D}$ & & & & 22.4 & & 0.00 & 0.00 \\
\hline HD 693 & $\mathrm{D}$ & 6173 & 11 & & 14.5 & 13.0 & -0.03 & -0.06 \\
\hline HD 9826 & $\mathrm{D}$ & 6119 & 4.12 & 0.12 & 33.7 & & 0.05 & -0.06 \\
\hline HD 10307 & $\mathrm{D}$ & 5776 & 4.13 & -0.12 & 19.7 & & -0.06 & 0.09 \\
\hline HD 10453 & $\mathrm{D}$ & 6368 & 3.96 & -0.46 & 13.0 & & 0.08 & -0.03 \\
\hline HD 13540 & $\mathrm{D}$ & 6301 & 4.12 & -0.43 & 13.9 & & 0.10 & -0.06 \\
\hline HD 16895 & $\mathrm{D}$ & 6228 & 4.27 & 0.01 & 21.0 & & -0.09 & -0.05 \\
\hline HD 22484 & $\mathrm{D}$ & 5915 & 4.03 & -0.16 & 24.1 & 23.5 & 0.06 & 0.07 \\
\hline HD 33632 & $\mathrm{D}$ & 5962 & 4.30 & -0.21 & 17.7 & & -0.01 & -0.04 \\
\hline HD 34411 & $\mathrm{D}$ & 5773 & 4.02 & -0.09 & 29.3 & 28.6 & 0.15 & 0.12 \\
\hline $\mathrm{HD} 3$ & $\mathrm{D}$ & 6202 & 3.83 & -0.38 & 14.5 & & 0.02 & -0.07 \\
\hline & $\mathrm{D}$ & 5805 & 4.29 & -0.18 & 16.9 & 18.9 & -0.08 & -0.04 \\
\hline HD 39833 & $\mathrm{D}$ & 5767 & 4.06 & -0.10 & 27.1 & 25.2 & 0.11 & 0.12 \\
\hline HD 43947 & $\mathrm{D}$ & 5859 & 4.23 & -0.30 & 16.7 & & 0.02 & -0.01 \\
\hline 575 & $\mathrm{D}$ & 5802 & 4.36 & -0.32 & 15.2 & & 0.01 & -0.03 \\
\hline & $\mathrm{D}$ & 6149 & 4.22 & -0.56 & 14.4 & & 0.21 & 0.05 \\
\hline 59380 & $\mathrm{D}$ & 6280 & 4.27 & -0.16 & 21.2 & & 0.06 & -0.01 \\
\hline HD 60319 & TD & 5867 & 4.24 & -0.80 & 7.9 & & 0.11 & 0.01 \\
\hline D 68146 & $\mathrm{D}$ & 6227 & 4.16 & -0.15 & 21.6 & & 0.04 & 0.06 \\
\hline 397 & $\mathrm{D}$ & 6243 & 4.28 & -0.32 & 16.4 & & 0.07 & 0.03 \\
\hline 332 & $\mathrm{D}$ & 6130 & 4.32 & 0.00 & 27.6 & & 0.03 & -0.09 \\
\hline $\mathrm{HD} 7$ & $\mathrm{D}$ & 6004 & 4.21 & -0.44 & 17.8 & & 0.18 & -0.01 \\
\hline $\mathrm{HD} 7$ & $\mathrm{D}$ & 6329 & 4.15 & 0.46 & 13.4 & & 0.10 & -0.03 \\
\hline 28 & $\mathrm{D}$ & 5874 & 4.06 & 0.09 & 27.6 & & 0.09 & 0.08 \\
\hline 328 & $\mathrm{D}$ & 6308 & 3.84 & -0.13 & 18.4 & & -0.06 & -0.12 \\
\hline & $\mathrm{D}$ & 5813 & 4.12 & & 28.6 & & -0.04 & -0.06 \\
\hline $\mathrm{HD}$ & $\mathrm{D}$ & 6051 & 4.36 & -0.17 & 15.2 & & -0.14 & -0.04 \\
\hline 889 & TD & 6020 & 4.15 & -0.25 & 18.9 & & 0.05 & 0.03 \\
\hline HD & $\mathrm{D}$ & 6063 & 4.10 & -0.06 & 30.6 & & 0.14 & 0.13 \\
\hline & $\mathrm{D}$ & 5731 & 4.16 & -0.07 & 24.2 & 23.5 & 0.01 & -0.02 \\
\hline 100180 & $\mathrm{D}$ & 5866 & 4.12 & -0.19 & 24.0 & & 0.11 & 0.05 \\
\hline & $\mathrm{D}$ & 6102 & 4.09 & & 11.2 & & 0.02 & 0.03 \\
\hline 303 & $\mathrm{D}$ & 5905 & 4.10 & -0.59 & 12.3 & & 0.12 & -0.07 \\
\hline & $\mathrm{D}$ & 5877 & 4.24 & -0.05 & 22.1 & 26.9 & -0.05 & 0.00 \\
\hline & $\mathrm{D}$ & 5866 & 4.03 & -0.02 & 24.4 & 26.3 & -0.07 & 0.01 \\
\hline 177 & $\mathrm{D}$ & 6061 & 3.93 & -0.63 & 9.7 & 9.2 & 0.03 & -0.02 \\
\hline 417 & $\mathrm{D}$ & 5925 & 4.30 & -0.05 & 27.3 & & 0.08 & -0.04 \\
\hline & $\mathrm{D}$ & 5935 & 4.32 & -0.46 & 13.8 & & 0.08 & 0.06 \\
\hline HD 162004 & $\mathrm{D}$ & 6059 & 4.12 & -0.26 & 19.1 & & 0.04 & 0.07 \\
\hline & $\mathrm{D}$ & 6298 & 4.15 & -0.02 & 19.3 & & -0.12 & -0.10 \\
\hline & $\mathrm{D}$ & 5888 & 4.26 & -0.30 & 18.1 & 17.3 & 0.06 & 0.03 \\
\hline & $\mathrm{D}$ & 6141 & 4.18 & -0.44 & 12.7 & & 0.02 & -0.05 \\
\hline HD 215648 & $\mathrm{D}$ & 6158 & 3.96 & -0.24 & 17.5 & 15.8 & -0.01 & -0.02 \\
\hline & $\mathrm{D}$ & 5923 & 3.74 & -0.15 & 19.5 & & -0.09 & -0.13 \\
\hline HD 222368 & $\mathrm{D}$ & 6178 & 4.08 & -0.13 & 19.0 & 19.0 & -0.05 & -0.03 \\
\hline
\end{tabular}

the $6362.35 \AA$ line as measured from the Moon spectrum observed in the same way as the program stars, we derive a solar zinc abundance of $\log \epsilon(\mathrm{Zn})=4.52$. This is considerably below the meteoritic $\mathrm{Zn}$ abundance of $4.67 \pm 0.04$
Table 2. Same as Table 1 but for stars from Nissen \& Schuster (1997) and $W_{a}$ is measured from the ESO NTT spectra.

\begin{tabular}{|c|c|c|c|c|c|c|c|c|}
\hline Star & Pop. & $\begin{array}{c}T_{\text {eff }} \\
\mathrm{K}\end{array}$ & $\log g$ & & $\begin{array}{r}W_{a} \\
\mathrm{~m} \AA\end{array}$ & $\begin{array}{c}W_{b} \\
\mathrm{~m} \AA\end{array}$ & {$\left[\frac{\mathrm{Zn}}{\mathrm{Fe}}\right]$} & {$\left[\frac{\mathrm{Ni}}{\mathrm{Fe}}\right]$} \\
\hline HD 17288 & TD & & 4.44 & -0.88 & 7.5 & & 0.25 & 0.00 \\
\hline & TD & & 4.24 & & 11.4 & & 0.20 & 0.03 \\
\hline G 005-040 & $\mathrm{H}$ & 5863 & 4.24 & -0.83 & 9.4 & & 0.26 & 0.02 \\
\hline CD-47 1087 & TD & 5734 & 4.38 & -0.76 & 9.6 & & 0.24 & -0.01 \\
\hline HD 22879 & TD & 5851 & 4.36 & -0.82 & 7.3 & 7.7 & 0.16 & -0.01 \\
\hline HD 24339 & TD & 5900 & 4.37 & -0.63 & 10.3 & & 0.14 & 0.02 \\
\hline HD 25704 & TD & 5886 & 4.33 & -0.85 & 6.6 & & 0.12 & -0.02 \\
\hline CD-57 1633 & $\mathrm{H}$ & 5933 & 4.26 & -0.90 & 4.1 & & -0.04 & -0.18 \\
\hline CD-45 3283 & $\mathrm{H}$ & 5672 & 4.57 & -0.83 & 4.1 & & 0.00 & -0.17 \\
\hline HD 60319 & TD & 5967 & 4.26 & 0.80 & 7.1 & & 0.11 & 0.01 \\
\hline HD 76932 & TD & 5914 & 4.23 & -0.85 & 6.7 & 7.0 & 0.12 & 0.01 \\
\hline G 046-031 & $\mathrm{H}$ & 6021 & 4.44 & -0.75 & 6.2 & & 0.04 & -0.12 \\
\hline HD 103723 & $\mathrm{H}$ & 6029 & 4.32 & -0.79 & 5.2 & & -0.02 & -0.11 \\
\hline 105004 & $\mathrm{H}$ & 5831 & 4.36 & -0.80 & 6.2 & & 0.06 & -0.06 \\
\hline HD 106516 & TD & 6269 & 4.51 & -0.61 & 7.6 & 5.8 & 0.05 & 0.00 \\
\hline GCRV 7547 & D & 6272 & 4.03 & -0.42 & 11.1 & & 0.01 & 0.00 \\
\hline HD 113679 & $\mathrm{H}$ & 5720 & 4.14 & -0.65 & 10.1 & & 0.18 & 0.03 \\
\hline 20559 & D & 5396 & 4.38 & -0.93 & 5.0 & & 0.12 & -0.01 \\
\hline HD 121004 & $\mathrm{H}$ & 5686 & 4.40 & -0.70 & 9.4 & & 0.17 & 0.00 \\
\hline
\end{tabular}

(Grevesse \& Sauval 1998). The same problem was encountered by Biémont \& Godefroid (1980), who derived a solar photospheric zinc abundance of $\log \epsilon(\mathrm{Zn})=4.54$ from the $\lambda 6362.35 \AA \mathrm{Zn}$ I line using the Holweger \& Müller (1974) model of the Sun. This difference between photospheric and meteoritic $\mathrm{Zn}$ abundances may well be due to an error in the $\log g f$ value of the $\mathrm{Zn}$ I line. However, when determining differential $\mathrm{Zn}$ abundances with respect to the Sun, i.e. $[\mathrm{Zn} / \mathrm{H}]$, using our solar photospheric abundance of $\log \epsilon(\mathrm{Zn})=4.52$ as a reference, the possible error in $\log g f$ cancels. The resulting values of $[\mathrm{Zn} / \mathrm{Fe}]$ are given in Tables 1 and 2 . The two tables also include values of $[\mathrm{Ni} / \mathrm{Fe}]$ as derived from more than $20 \mathrm{Ni}$ I lines (see Chen et al. 2000; Nissen \& Schuster 1997).

\section{Errors of the derived abundance ratios}

The uncertainties of the model parameters are estimated to be $\pm 100 \mathrm{~K}$ in temperature, \pm 0.15 dex in gravity, \pm 0.1 dex in metallicity and $\pm 0.3 \mathrm{~km} \mathrm{~s}^{-1}$ in microturbulence. The dependence of the derived $[\mathrm{Fe} / \mathrm{H}]$ and $[\mathrm{Zn} / \mathrm{Fe}]$ abundance ratios on the stellar parameters is calculated by altering temperature, gravity, metallicity and microturbulence for HD 60319, HD 69897 and the Sun, representing most of our metallicity range. As seen from Table 3, $[\mathrm{Zn} / \mathrm{Fe}]$ is most sensitive to gravity, but the combined error is small - less than $0.06 \mathrm{dex}$ if the individual errors are added quadratically. We also checked that the derived $[\mathrm{Zn} / \mathrm{Fe}]$ ratio is only marginally affected $(<0.02 \mathrm{dex})$, when alpha-element enhanced models $([\alpha / \mathrm{Fe}]=+0.4, \alpha=\mathrm{O}$, $\mathrm{Ne}, \mathrm{Mg}, \mathrm{Si}, \mathrm{S}, \mathrm{Ca}$, and $\mathrm{Ti}$ ) are used instead of models with $[\alpha / \mathrm{Fe}]=0.0$. Taking into account the uncertainty of the measured equivalent width of the $\lambda 6362.35 \AA \mathrm{Zn}$ I line $( \pm 1.3 \mathrm{~m} \AA)$ we then estimate a total error of about $0.07 \mathrm{dex}$ for $[\mathrm{Zn} / \mathrm{Fe}]$ and $[\mathrm{Fe} / \mathrm{H}]$. A similar error is estimated for $[\mathrm{Zn} / \mathrm{Ni}]$ and $[\mathrm{Ni} / \mathrm{H}]$. 
Table 3. Dependence of $[\mathrm{Fe} / \mathrm{H}]$ and $[\mathrm{Zn} / \mathrm{Fe}]$ on stellar parameter variations for two representative stars and the Sun. $\mathrm{Zn}$ abundances are based on the $\lambda 6362.35 \mathrm{Zn}$ I line and $\mathrm{Fe}$ abundances on $\mathrm{Fe}$ II lines.

\begin{tabular}{|c|c|c|c|c|c|c|}
\hline & \multicolumn{2}{|c|}{ HD $60319^{1}$} & \multicolumn{2}{|c|}{ HD $69897^{2}$} & \multicolumn{2}{|c|}{$\operatorname{Sun}^{3}$} \\
\hline & $\Delta\left[\frac{\mathrm{Fe}}{\mathrm{H}}\right]$ & $\Delta\left[\frac{\mathrm{Zn}}{\mathrm{Fe}}\right]$ & $\Delta\left[\frac{\mathrm{Fe}}{\mathrm{H}}\right]$ & $\Delta\left[\frac{\mathrm{Zn}}{\mathrm{Fe}}\right]$ & $\Delta\left[\frac{\mathrm{Fe}}{\mathrm{H}}\right]$ & $\Delta\left[\frac{\mathrm{Zn}}{\mathrm{Fe}}\right]$ \\
\hline$\Delta T_{\text {eff }}=100 \mathrm{~K}$ & -0.01 & 0.02 & -0.01 & 0.03 & -0.04 & 0.02 \\
\hline$\Delta \log g=0.15$ & 0.06 & -0.03 & 0.06 & -0.03 & 0.06 & -0.03 \\
\hline$\Delta[\mathrm{Fe} / \mathrm{H}]=0.1$ & 0.01 & -0.01 & 0.01 & -0.01 & 0.03 & -0.01 \\
\hline$\Delta \xi_{\mathrm{t}}=0.3 \mathrm{~km} \mathrm{~s}^{-1}$ & -0.02 & 0.02 & -0.04 & 0.03 & -0.03 & 0.01 \\
\hline$E_{\gamma}=1.0 \rightarrow 2.0$ & & 0.00 & & 0.00 & & -0.02 \\
\hline
\end{tabular}

1) HD 60319: $T_{\text {eff }}=5867 \mathrm{~K}, \log g=4.24,[\mathrm{Fe} / \mathrm{H}]=-0.8, \xi_{\mathrm{t}}=1.6$.

2) HD 69897: $T_{\mathrm{eff}}=6243 \mathrm{~K}, \log g=4.28,[\mathrm{Fe} / \mathrm{H}]=-0.3, \xi_{\mathrm{t}}=2.0$.

3) The Sun: $T_{\text {eff }}=5870 \mathrm{~K}, \log g=4.44,[\mathrm{Fe} / \mathrm{H}]=0.0, \xi_{\mathrm{t}}=1.15$.

In calculating zinc abundances from the $6362.35 \AA$ $\mathrm{Zn}$ I line we adopted the Unsöld (1955) approximation to the Van der Waals interaction constant with an enhancement factor $E_{\gamma}=1.5$. As seen from Table 3, the derived zinc abundance depends only slightly on the value of $E_{\gamma}$; the effect of increasing the enhancement factor from 1.0 to 2.0 is less than $0.03 \mathrm{dex}$ even in the most metal rich stars. This is in stark contrast to the effect of $E_{\gamma}$ on the stronger $\mathrm{Zn}$ I lines at 4722.2 and $4810.5 \AA$, for which an increase of $E_{\gamma}$ from 1.0 to 2.0 leads to a decrease of the derived $\mathrm{Zn}$ abundance for the Sun with about $0.13 \mathrm{dex}$, whereas the effect for the metal-poor disk stars is a decrease of 0.02 dex only. Hence, $[\mathrm{Zn} / \mathrm{Fe}]$ in metal-poor disk stars becomes very sensitive to the adopted value of $E_{\gamma}$ if the stronger $\mathrm{Zn}$ I lines are used. This is the main reason that we have avoided these lines in the present paper.

As mentioned in Sect. 2 the $6362.35 \AA \mathrm{Zn}$ I line lays in the midst of a $\sim 15 \AA$ broad and shallow absorption feature with a central depth of a few percent identified by Michell \& and Mohler (1965) as due to a Ca I auto-ionization line. The equivalent width of the $\mathrm{Zn}$ I line was measured relative to the local apparent continuum but the small contribution of the Ca I autoionization line to the absorption coefficient has been neglected in our analysis. We have investigated the effect of this autoionization line on the derived $\mathrm{Zn}$ abundance by synthesizing the solar flux spectrum in the spectral region 6356-6368. In order to fit the large width, we adopt a very high radiation damping constant $(1.4 \mathrm{E}+12)$ of the Ca I auto-ionization line and vary its (unknown) $\log g f$ value so that the right equivalent width is obtained. In the case of the Sun, the effect of including the $\mathrm{Ca}$ I auto-ionization line is to increase the $\mathrm{Zn}$ abundance by +0.03 dex, i.e. from $\log \epsilon(\mathrm{Zn})=4.52$ to 4.55 . For a typical metal-poor thick disk star, HD 60319, with $[\mathrm{Fe} / \mathrm{H}]=-0.80$ and $[\mathrm{Ca} / \mathrm{Fe}] \sim+0.2$, the correction of the $\mathrm{Zn}$ abundance is of the order of +0.01 dex. Differentially with respect to the Sun, the largest effect of including the opacity contribution from the $\mathrm{Ca}$ I auto-ionization line would then be a decrease of $[\mathrm{Zn} / \mathrm{Fe}]$ for the most metal-poor stars by about 0.02 dex. This is quite negligible compared with the other error sources.

As described in Sect. 3 we have derived the abundances on the basis of plane parallel, homogeneous (1D) model atmospheres and the assumption of LTE. Asplund et al. (1999) have shown that there may be significant effects from using three-dimensional (3D) hydrodynamical models instead, especially for metal-poor stars and for lines formed in the upper part of the atmosphere such as molecular lines (Asplund \& García Pérez 2001). For lines formed deep in the atmosphere, such as the $\mathrm{Zn}$ Iand $\mathrm{Fe}$ II lines which we have used, the 3D effects are, however, small (Nissen et al. 2004) and go in the same direction. Hence, we don't expect that the derived $[\mathrm{Zn} / \mathrm{Fe}]$ is subject to any significant $3 \mathrm{D}$ corrections.

Regarding non-LTE effects on $[\mathrm{Zn} / \mathrm{Fe}]$ we first note that for the Chen et al. sample the iron abundances are based on Fe II lines for which departures from LTE are small according to the computations of Thévenin \& Idiart (1999). For the Nissen $\&$ Schuster (1997) stars we adopted iron abundances based on $\mathrm{Fe}_{\mathrm{I}}$ lines and determined the gravities by requiring that $\mathrm{Fe}_{\mathrm{I}}$ and $\mathrm{Fe}_{\mathrm{II}}$ lines should provide the same Fe abundance. Hence, one might expect that the derived iron abundances are too low due to an overionization of $\mathrm{Fe}_{\mathrm{I}}$ as predicted by Thévenin \& Idiart (1999) for metal-poor stars. However, as discussed in Sect. 3, gravities derived from Hipparcos parallaxes for a subsample of the Nissen \& Schuster stars agree very well with the spectroscopic gravities. Hence, we conclude that the derived iron abundances are not significantly different from the true Fe abundances. This should not be taken as evidence against the predicted over-ionization of Fe for metal-poor stars. Rather, it reflects the way the $g f$-values were determined by Nissen $\&$ Schuster, namely by an "inverted" abundance analysis of the two bright stars, HD 22879 and HD 76932, with their parameters and abundances taken from Edvardsson et al. (1993).

It is more unclear if there are non-LTE effects on the derived $\mathrm{Zn}$ abundances. With an ionization potential, $\chi_{\text {ion }}(\mathrm{Zn} \mathrm{I})=$ $9.39 \mathrm{eV}$, there are approximately equal numbers of neutral and ionized zinc atoms at the temperatures and electron pressures of the line forming regions in the atmospheres of our stars. Hence, an overionization of $\mathrm{Zn}$ I relative to LTE, as is the case for Fe I (Thévenin \& Idiart 1999), would lead to an underestimate of the abundance of $\mathrm{Zn}$ in the LTE analysis of $\mathrm{Zn}$ I lines. However, such an over-ionization effect on $\mathrm{Zn}$ I is likely to be smaller than the equivalent effect for Fe I, since $N_{\mathrm{Zn} \text { I }} \sim N_{\mathrm{Zn} \text { II }}$ whereas $N_{\mathrm{Fe} \text { I }} \ll N_{\mathrm{Fe} \text { II }}$, where $N$ denotes the number density of the atoms and ions. Furthermore, we note that the $6362.35 \AA$ $\mathrm{Zn}$ I line, on which our $\mathrm{Zn}$ abundances is based, is a weak, high excitation potential $\left(\chi_{\mathrm{exc}}=5.79 \mathrm{eV}\right)$ line formed deep in the atmosphere, where departures from LTE tend to be small due to the high density. Nevertheless, it would be very desirable to perform a thorough study of non-LTE effects on the determination of $\mathrm{Zn}$ abundances.

\section{Results and discussion}

Following Fuhrmann $(1998,2000)$ we have used the total space velocity, $V_{\text {tot }}=\left(U^{2}+V^{2}+W^{2}\right)^{\frac{1}{2}}$, with respect to the Local Standard of Rest (LSR) to classify the stars in three main populations: i) thin disk stars with $V_{\text {tot }}<85 \mathrm{~km} \mathrm{~s}^{-1}$; ii) thick disk stars with $85<V_{\text {tot }}<180 \mathrm{~km} \mathrm{~s}^{-1}$; and iii) halo stars with $V_{\text {tot }}>180 \mathrm{~km} \mathrm{~s}^{-1}$. For stars in Table 1 , the $U, V, W$ velocity components with respect to the LSR are taken from 


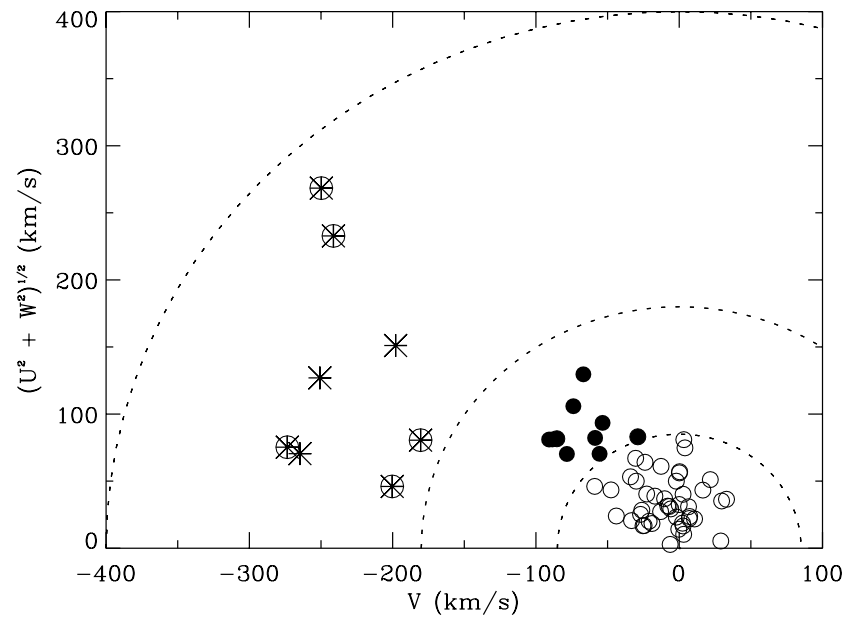

Fig. 4. Toomre diagram for stars in Tables 1 and 2. Open circles refer to thin disk stars $\left(V_{\text {tot }}<85 \mathrm{~km} \mathrm{~s}^{-1}\right)$, filled circles to thick disk stars $\left(85<V_{\text {tot }}<180 \mathrm{~km} \mathrm{~s}^{-1}\right)$, and asterisks to halo stars $\left(V_{\text {tot }}>\right.$ $180 \mathrm{~km} \mathrm{~s}^{-1}$ ). Alpha-poor halo stars are encircled. The dotted circles refer to total space velocities of 85,180 and $400 \mathrm{~km} \mathrm{~s}^{-1}$ with respect to the Local Standard of Rest.

Chen et al. (2000, 2002). For stars in Table 2 we have updated the velocity components calculated by Nissen \& Schuster (1997) using Hipparcos (ESA 1997) or Tycho-2 (Høg et al. 2000) proper motions and adopting the most recent values for the solar motion with respect to the $\operatorname{LSR}\left(\left(U_{\odot}, V_{\odot}, W_{\odot}\right)=\right.$ $\left(-10.0,+5.2,+7.2 \mathrm{~km} \mathrm{~s}^{-1}\right)^{1}$ (Dehnen \& Binney 1998). The changes with respect to Nissen \& Schuster are relatively small except for one star, GCRV 7547 (CD-39 7674) that was classified as a halo star with an unusually high metallicity $([\mathrm{Fe} / \mathrm{H}]=$ -0.42) by Nissen \& Schuster. With the new proper motion values from the Tycho catalogue, it turns out to be an ordinary thin disk star with velocity components $(U, V, W)=(35,30$, 7) $\mathrm{km} \mathrm{s}^{-1}$.

The large majority of stars in Table 1 have thin disk kinematics, whereas those in Table 2 are mainly thick disk or halo stars. As seen from the Toomre diagram in Fig. 4, the thick disk and the halo stars are very well separated in $V$ in accordance with the way the two groups were selected by Nissen $\&$ Schuster (1997). The thin and thick disk stars have a slight overlap in $V$, but all thick disk stars except one have a rotational lag in the range $-100<V<-50 \mathrm{~km} \mathrm{~s}^{-1}$, whereas all thin disk stars except one have $V>-50 \mathrm{~km} \mathrm{~s}^{-1}$.

Figure 5 shows $[\mathrm{Zn} / \mathrm{Fe}]$ versus $[\mathrm{Fe} / \mathrm{H}]$ with different symbols for the various populations. Looking first at the thin disk stars, we see a slight increasing trend of $[\mathrm{Zn} / \mathrm{Fe}]$ with decreasing metallicity; a straight line least squares fit to the disk star data gives:

$$
[\mathrm{Zn} / \mathrm{Fe}]=-0.01( \pm 0.02)-0.16( \pm 0.05) \cdot[\mathrm{Fe} / \mathrm{H}]
$$

with a reduced chi-square, $\chi_{\text {red }}^{2}=1.11$, when the estimated error of $0.07 \mathrm{dex}$ on $[\mathrm{Zn} / \mathrm{Fe}]$ and $[\mathrm{Fe} / \mathrm{H}]$ is adopted. This relation corresponds to $[\mathrm{Zn} / \mathrm{Fe}] \simeq+0.1$ for a thin disk metallicity of $[\mathrm{Fe} / \mathrm{H}]=-0.6$, and the relation agrees quite well with the

\footnotetext{
${ }^{1}$ In the present work, $U$ is defined to be positive in the anticentre direction.
}

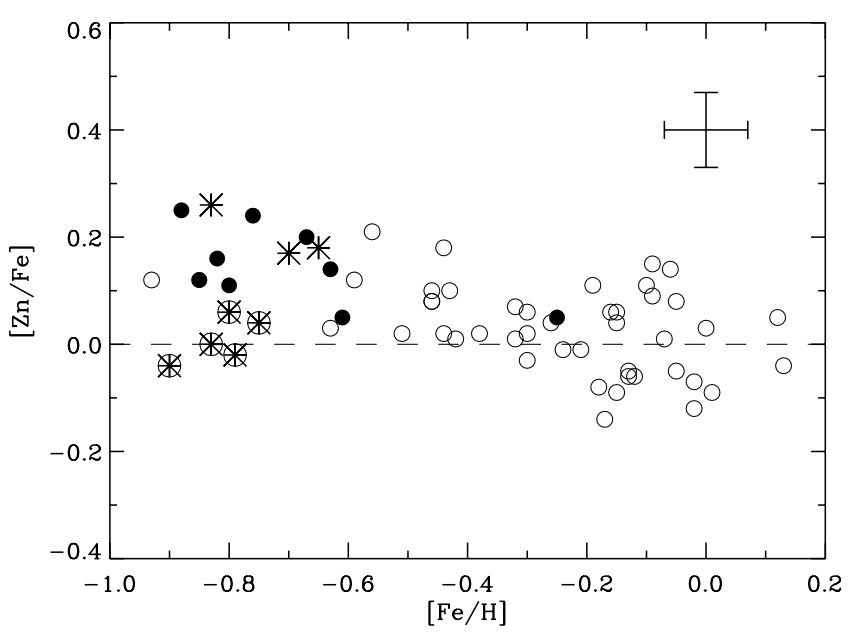

Fig. 5. $[\mathrm{Zn} / \mathrm{Fe}]$ versus $[\mathrm{Fe} / \mathrm{H}]$. The various symbols refer to thin disk stars (open circles), thick disk stars (filled circles), and halo stars (asterisks) with alpha-poor halo stars encircled.

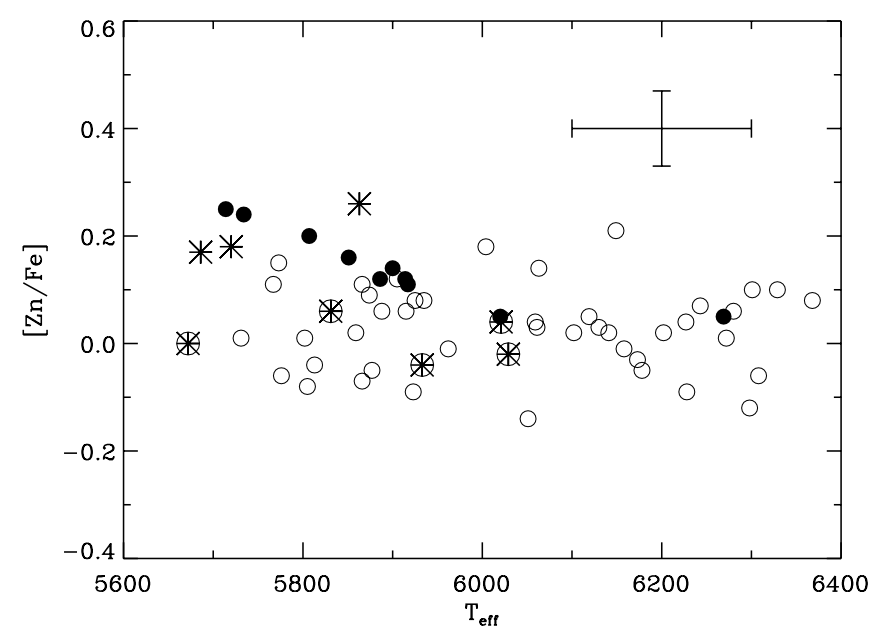

Fig. 6. $[\mathrm{Zn} / \mathrm{Fe}]$ versus $T_{\text {eff. }}$ The symbols are the same as those in Fig. 5.

trend of $[\mathrm{Zn} / \mathrm{Fe}]$ versus $[\mathrm{Fe} / \mathrm{H}]$ found by Reddy et al. (2003) and Bensby et al. (2003) for thin disk stars.

Bensby et al. (2003) have found evidence of a difference of about 0.1 dex in $[\mathrm{Zn} / \mathrm{Fe}]$ between thick and thin disk stars in the metallicity range $-0.6<[\mathrm{Fe} / \mathrm{H}]<-0.3$. As seen from Fig. 5, we have no thick disk stars in this metallicity range. Our ten thick disk stars in the metallicity range $-0.9<[\mathrm{Fe} / \mathrm{H}]<-0.6$ have a mean $[\mathrm{Zn} / \mathrm{Fe}]$ of $0.15 \mathrm{dex}$, which is only slightly higher than the value predicted from the thin disk relation given above. The explanation may be that our thick disk stars lie in a metallicity range, $[\mathrm{Fe} / \mathrm{H}]<-0.6$, where the thick and the thin disk are not chemically well separated. The same is seen for e.g. the ratio between alpha-capture elements and $\mathrm{Fe}$, i.e. $[\alpha / \mathrm{Fe}]$. In the metallicity range $-0.6<[\mathrm{Fe} / \mathrm{H}]<-0.3[\alpha / \mathrm{Fe}]$ is different for thick and thin disk stars (Bensby et al. 2003, 2004), whereas the two populations start to merge together in $[\alpha / \mathrm{Fe}]$ for $[\mathrm{Fe} / \mathrm{H}] \simeq-0.7$.

Among the eight halo stars shown in Fig. 5, three have an enhanced $\mathrm{Zn} / \mathrm{Fe}$ ratio at the same level as the thick disk stars, but the other five have a solar $\mathrm{Zn} / \mathrm{Fe}$ ratio. As shown in Figs. 6 and 7, the eight halo stars and the ten thick disk 


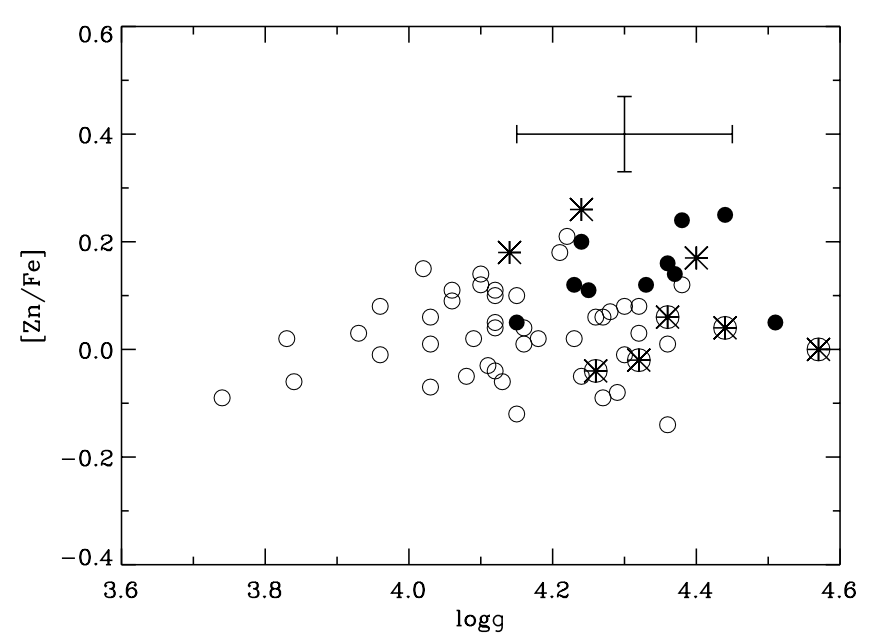

Fig. 7. $[\mathrm{Zn} / \mathrm{Fe}]$ versus $\log g$. The symbols are the same as those in Fig. 5.

stars have similar temperatures and gravities, and there is no significant trend of $[\mathrm{Zn} / \mathrm{Fe}]$ with the stellar parameters. Thus, the scatter of $[\mathrm{Zn} / \mathrm{Fe}]$ among the halo stars seems to be real. Furthermore, the five halo stars with low $[\mathrm{Zn} / \mathrm{Fe}]$ are among the group of so-called alpha-poor halo stars that were found by Nissen \& Schuster (1997) to have a solar-like ratio between the alpha-capture elements (O, Mg, Si, Ca and Ti) and Fe. Hence, $\mathrm{Zn}$ tends to follow the alpha-capture elements in two respects: by being overabundant in metal-poor disk stars and by having a solar-like $[\mathrm{Zn} / \mathrm{Fe}]$ for the alpha-poor halo stars. The amplitude of the $[\mathrm{Zn} / \mathrm{Fe}]$ variations is, however, smaller than in the case of $[\mathrm{O} / \mathrm{Fe}]$ and $[\mathrm{Mg} / \mathrm{Fe}]$, i.e. $\sim 0.15$ dex instead of $\sim 0.3 \mathrm{dex}$.

Nissen \& Schuster (1997) also found the alpha-poor halo stars to have unusual low abundances of $\mathrm{Na}$ and $\mathrm{Ni}$ with respect to $\mathrm{Fe}$. $[\mathrm{Na} / \mathrm{Fe}]$ varies from -0.4 to 0.0 and $[\mathrm{Ni} / \mathrm{Fe}]$ varies from -0.2 to 0.0 with a tight correlation between the two ratios. As discussed by Nissen (2004) recent abundance studies of giant stars in dwarf spheroidal galaxies (Shetrone et al. 2003) show that these stars also have underabundant values of $[\mathrm{Na} / \mathrm{Fe}]$ and $[\mathrm{Ni} / \mathrm{Fe}]$ and fit the $[\mathrm{Na} / \mathrm{Fe}]-[\mathrm{Ni} / \mathrm{Fe}]$ relation of alpha-poor halo stars very well. This supports the suggestion of Nissen \& Schuster (1997) that the alpha-poor halo stars have been accreted from dwarf galaxies with a different star formation history and/or initial mass function than the Milky Way. The deficiency of $\mathrm{Na}$ and $\mathrm{Ni}$ may be connected to the fact that the yields of both $\mathrm{Na}$ and the dominant $\mathrm{Ni}$ isotope $\left({ }^{58} \mathrm{Ni}\right)$ depend upon the neutron excess (Thielemann et al. 1990). The deficiency of $\mathrm{Zn}$ in the alpha-poor halo stars may then be explained by the fact that the production of $\mathrm{Zn}$ in type II SNe also depends on the neutron excess (Timmes et al. 1995). Indeed, Fig. 8 shows that the alpha-poor halo stars do not stand out in a plot of $[\mathrm{Zn} / \mathrm{Ni}]$ versus $[\mathrm{Ni} / \mathrm{H}]$.

The $\lambda 6362.25 \AA \mathrm{Zn}$ I line is too weak to be used for deriving $\mathrm{Zn}$ abundances in stars with $[\mathrm{Fe} / \mathrm{H}]<-1.0$. For such stars the $\lambda \lambda 4722.16,4810.54 \AA \mathrm{Zn}$ I lines are, however, quite ideal having equivalent widths ranging from a few $\mathrm{m} \AA$ to about $50 \mathrm{~m} \AA$ when $[\mathrm{Fe} / \mathrm{H}]$ increases from -2.5 to -0.8 . Figure 9 includes such $[\mathrm{Zn} / \mathrm{Fe}]$ data for 29 stars from Nissen et al. (2004).

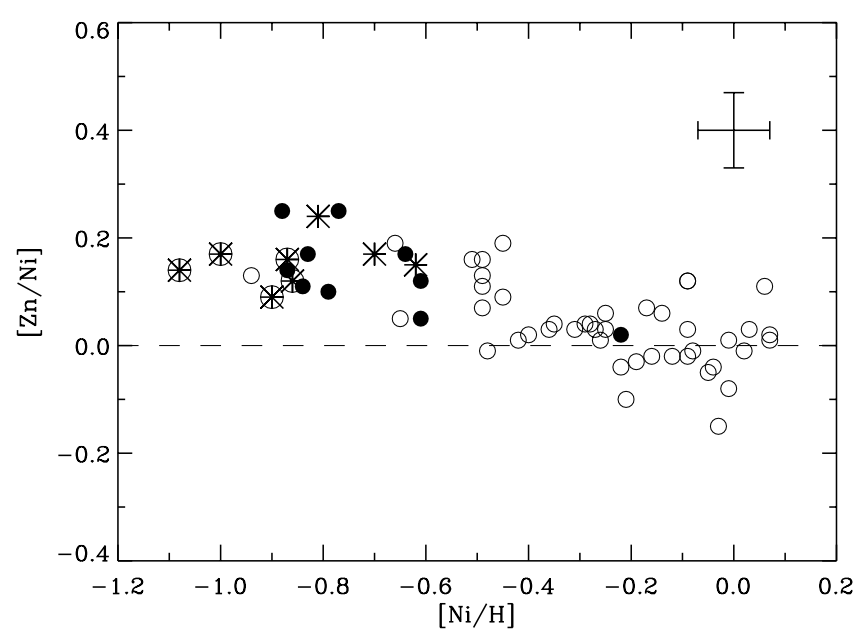

Fig. 8. $[\mathrm{Zn} / \mathrm{Ni}]$ versus $[\mathrm{Ni} / \mathrm{H}]$ with the same symbols as in Fig. 5.

Three of the stars, HD 103723, HD 105004 and HD 121004 are in common with the present paper; their symbols are connected with straight lines in Fig. 9. As seen, there is a reasonable good agreement between the two sets of data. The mean difference in $[\mathrm{Zn} / \mathrm{Fe}]$ (Nissen et al.; present paper) is -0.06 dex suggesting perhaps a slight offset of the data based on the $\lambda \lambda 4722.16$, $4810.54 \AA$ lines, which may occur because the lines are quite strong in the Sun causing $[\mathrm{Zn} / \mathrm{Fe}]$ to be sensitive to the adopted value of the damping constant as discussed in Sect. 4.

From Fig. 9 it is seen that although the overall $[\mathrm{Zn} / \mathrm{Fe}]$ trend is quite flat over the metallicity range $-2.5<[\mathrm{Fe} / \mathrm{H}]<+0.2$, subtle deviations of $[\mathrm{Zn} / \mathrm{Fe}]$ from zero seem to occur. The most metal-poor halo stars have $[\mathrm{Zn} / \mathrm{Fe}] \sim+0.1$ and metal-poor thin disk and thick disk stars have $[\mathrm{Zn} / \mathrm{Fe}] \sim+0.10$ to +0.15 . One might argue that such small deviations could be due to errors in the analysis, because non-LTE effects were not taken into account. On the other hand, it seems very difficult to explain the systematic difference of about 0.15 dex in $[\mathrm{Zn} / \mathrm{Fe}]$ between thick disk stars and alpha-poor halo stars at the same metallicity as a non-LTE effect. We are here comparing abundances of stars with nearly the same atmospheric parameters, $T_{\text {eff }}, \log g$ and $[\mathrm{Fe} / \mathrm{H}]$. Hence, any non-LTE effects are expected to cancel when determining the difference in $[\mathrm{Zn} / \mathrm{Fe}]$.

We therefore think that the small deviations of $[\mathrm{Zn} / \mathrm{Fe}]$ from zero are real and contain important information about the complicated nucleosynthesis of $\mathrm{Zn}$. For the most metal-poor halo stars Cayrel et al. (2004) also find a slight overabundance of $[\mathrm{Zn} / \mathrm{Fe}]$ at $[\mathrm{Fe} / \mathrm{H}]=-2.5$ increasing to $[\mathrm{Zn} / \mathrm{Fe}] \sim+0.5$ at $[\mathrm{Fe} / \mathrm{H}]=-4.0$. As discussed by Umeda \& Nomoto (2002) this trend and other non-solar abundance ratios for the iron-peak elements may be explained with high-energy (hypernovae) models for core collapse explosions of massive Population III stars. In the case of the thick disk and metal-poor thin disk stars, the overabundance of $\mathrm{Zn} / \mathrm{Fe}$ indicates that there is a source of zinc production in addition to the weak $s$-process and alpha-rich freezeout in type II SNe. This additional contribution may be due to zinc production in the neutrino-powered wind of 10 to 20 solar mass $\mathrm{SNe}$ as suggested by Hoffman et al. (1996), essentially at the same site as proposed for the 


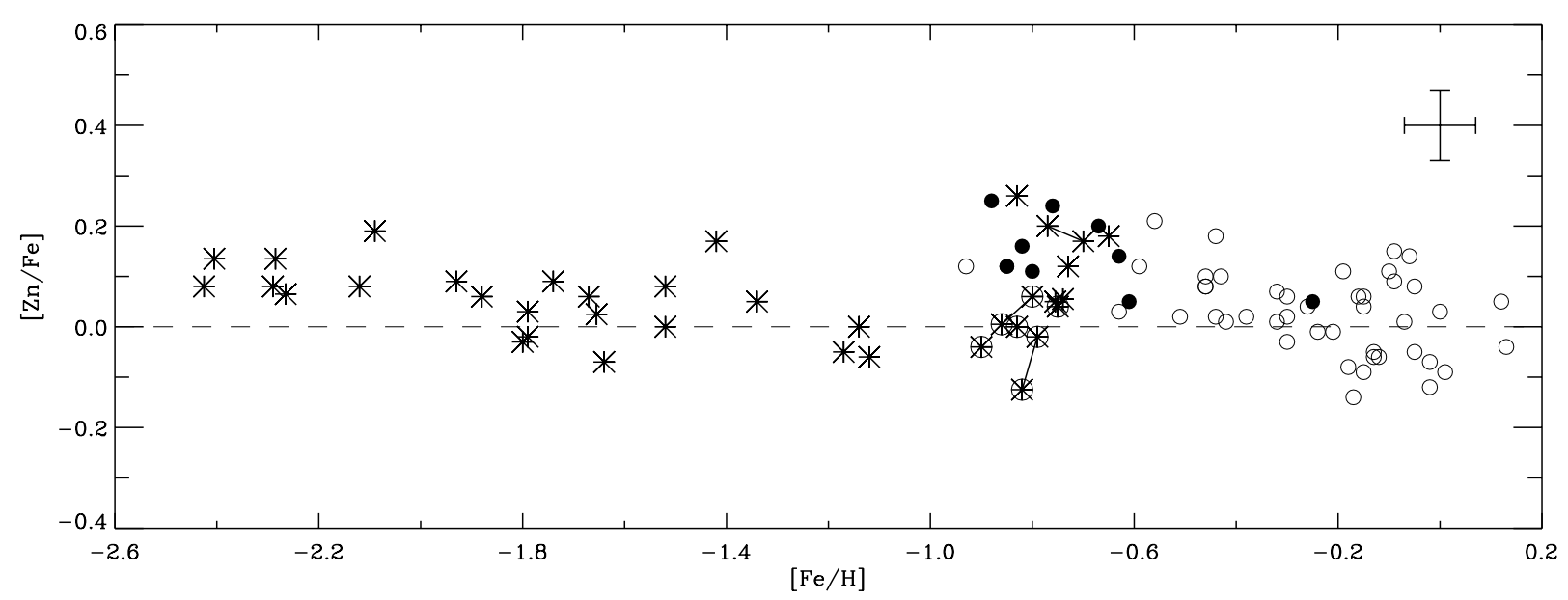

Fig. 9. $[\mathrm{Zn} / \mathrm{Fe}]$ versus $[\mathrm{Fe} / \mathrm{H}]$ with the same symbols as in Fig. 5 and including $[\mathrm{Zn} / \mathrm{Fe}]$ for metal-poor halo stars as derived from the $\lambda \lambda 4722.16$, 4810.54 $\AA \mathrm{Zn}$ I lines by Nissen et al. (2004). Three stars that are in common between the present work and Nissen et al. have been connected by straight lines.

$r$-process (Woosley et al. 1994). In this connection, we note that the $r$-process elements are also known to be overabundant with respect to $\mathrm{Fe}$ in thick disk and metal-poor thin disk stars (Mashonkina et al. 2003). Furthermore, it is interesting that it is the ${ }^{64} \mathrm{Zn}$ isotope, which is produced in the neutrino-powered wind according to Hoffman et al. (1996). ${ }^{64} \mathrm{Zn}$ is the dominant isotope in the solar system (Anders \& Grevesse 1989) but is underproduced by a factor of three in traditional calculations of nucleosynthesis of $\mathrm{Zn}$ in type II SNe (Timmes et al. 1995, Fig. 3).

As seen from Fig. 9, the halo stars in the metallicity range $-1.8<[\mathrm{Fe} / \mathrm{H}]<-1.0$ have $[\mathrm{Zn} / \mathrm{Fe}] \sim 0.0$. They form a sequence in the figure that fit well to the alpha-poor halo stars at $[\mathrm{Fe} / \mathrm{H}] \sim-0.8$. The thick disk and the halo stars at $[\mathrm{Fe} / \mathrm{H}] \sim-0.8$ with $[\mathrm{Zn} / \mathrm{Fe}] \sim+0.15$ are apparently subject to a different evolution in $[\mathrm{Zn} / \mathrm{Fe}]$. The explanation may be that the Galactic halo consists of two components as also suggested by Gratton et al. (2003): a dissipative component with high $[\mathrm{Zn} / \mathrm{Fe}]$ and $[\alpha / \mathrm{Fe}]$ connected to the thick disk and an accreted component with low $[\mathrm{Zn} / \mathrm{Fe}]$ and $[\alpha / \mathrm{Fe}]$.

Although these possible variations of $[\mathrm{Zn} / \mathrm{Fe}]$ as a function of $[\mathrm{Fe} / \mathrm{H}]$ are of importance when interpreting abundance data for DLAs, we note that the amplitude of $[\mathrm{Zn} / \mathrm{Fe}]$ is smaller than in the case of $[\alpha / \mathrm{Fe}]$. Sulphur is of particular interest in this connection, because $\mathrm{S}$ like $\mathrm{Zn}$ is practically undepleted onto dust so that the observed $\mathrm{S} / \mathrm{Zn}$ interstellar gas ratio in DLAs equals the intrinsic abundance ratio between the two elements. As shown by Nissen et al. (2004), the overabundance of $\mathrm{S}$ in halo stars corresponds to $[\mathrm{S} / \mathrm{Fe}] \sim+0.3$ to +0.4 dex. Hence, when plotting $[\mathrm{S} / \mathrm{Zn}]$ versus $[\mathrm{Zn} / \mathrm{H}]$ we still get a trend that looks like [S/Fe] versus [Fe/H] (see Nissen et al. 2004, Figs. 6 and 8 ). This suggests that $[\mathrm{S} / \mathrm{Zn}]$ may be used as a "chemical clock" to date the star formation process at high $z$ albeit with a lower sensitivity than $[\mathrm{S} / \mathrm{Fe}]$. As discussed by Fenner et al. (2004), a better understanding of the nucleosynthesis of $\mathrm{Zn}$ is, however, needed in order to derive the past history of star formation in DLAs from the observed $\mathrm{S} / \mathrm{Zn}$ ratio.

\section{Conclusions}

We have determined $\mathrm{Zn}$ abundances for 62 dwarf stars with metallicities in the range $-1.0<[\mathrm{Fe} / \mathrm{H}]<+0.2$. The abundances are based on equivalent widths of the weak $\lambda 6362.35 \AA$ $\mathrm{Zn}$ I line and are relatively insensitive to possible errors in the atmospheric parameters and the damping constant of the line; thus they are more reliable than $\mathrm{Zn}$ abundances based on the stronger $\lambda \lambda 4722.16,4810.54 \AA \mathrm{Zn}$ I lines, which have been applied in other studies of $\mathrm{Zn}$ abundances.

The stars were grouped into thin disk, thick disk and halo populations according to their kinematics. It is found that $[\mathrm{Zn} / \mathrm{Fe}]$ in thin disk stars shows a slight increasing trend with decreasing metallicity reaching a value of $[\mathrm{Zn} / \mathrm{Fe}] \sim+0.1$ at $[\mathrm{Fe} / \mathrm{H}]=-0.6$ in agreement with Reddy et al. (2003) and Bensby et al. (2003). Ten thick disk stars in the metallicity range $-0.9<[\mathrm{Fe} / \mathrm{H}]<-0.6$ have an average $[\mathrm{Zn} / \mathrm{Fe}]=$ $+0.15 \mathrm{dex}$, whereas five alpha-poor halo stars in the same metallicity range have $[\mathrm{Zn} / \mathrm{Fe}] \simeq 0.0 \mathrm{dex}$. Interestingly, the same five stars are also underabundant in Ni having an average $[\mathrm{Ni} / \mathrm{Fe}] \simeq-0.13$.

These results suggest that $\mathrm{Zn}$ is not an exact tracer of Fe as often assumed in abundance studies of DLA systems. The overabundance of $\mathrm{Zn} / \mathrm{Fe}$ in metal-poor thin and thick disk stars may be explained by $\mathrm{Zn}$ production in the neutrino-powered wind of 10 to 20 solar mass $\mathrm{SNe}$ as suggested by Hoffman et al. (1996). The deviations of $[\mathrm{Zn} / \mathrm{Fe}]$ from zero are, however, considerably smaller than in the case of $[\mathrm{O} / \mathrm{Fe}],[\mathrm{Mg} / \mathrm{Fe}]$ and $[\mathrm{S} / \mathrm{Fe}] ;[\mathrm{S} / \mathrm{Zn}]$ versus $[\mathrm{Zn} / \mathrm{H}]$ for DLA systems may therefore still be used to obtain information on the star formation history in these objects if the nucleosynthesis of zinc can be better understood.

In order to improve on the study of the $[\mathrm{Zn} / \mathrm{Fe}]-[\mathrm{Fe} / \mathrm{H}]$ trend for Galactic stars even more accurate data for the equivalent width of $\mathrm{Zn}$ lines should be obtained in larger samples of disk and halo stars. At the same time a thorough study of nonLTE effects on the determination of $\mathrm{Zn}$ abundances is needed, 
as well as further studies of the nucleosynthesis of zinc in various types of objects.

Acknowledgements. This work is partly supported by the National Natural Science Foundation of China and grant NKBRSF G1999075406, and by the Danish Natural Science Research Council, grant 21-01-0523.

\section{References}

Alonso, A., Arribas, S., \& Martínez-Roger, C. 1996, A\&A, 313, 873 Anders, E., \& Grevesse, N. 1989, Geochim. Cosmochim. Acta, 53, 197

Asplund, M., \& García Pérez, A. E. 2001, A\&A, 372, 601

Asplund, M., Gustafsson, B., Kiselman, D., \& Eriksson, K. 1997, A\&A, 318, 521

Asplund, M., Nordlund, A., Trampedach, R., \& Stein, R. F. 1999, A\&A, 346, L17

Bensby, T., Feltzing, S., \& Lundström, I. 2003, A\&A, 410, 527

Bensby, T., Feltzing, S., \& Lundström, I. 2004, A\&A, 415, 155

Biémont, E., \& Godefroid, M. 1980, A\&A, 84, 361

Cayrel, R., Depagne, E., Spite, M., et al. 2004, A\&A, 416, 1117

Centurión, M., Bonifacio, P., Molaro, P., \& Vladilo, G. 2000, ApJ, 536,540

Chen, Y. Q., Nissen, P. E., Zhao, G., Zhang, H. W., \& Benoni, T. 2000, A\&AS, 141, 491

Chen, Y. Q., Nissen, P. E., Zhao, G., \& Asplund, M. 2002, A\&A, 390, 225

Dehnen, W., \& Binney, J. J. 1998, MNRAS, 298, 387

Edvardsson, B., Andersen, J., Gustafsson, B., et al. 1993, A\&A, 275, 101

ESA 1997, The Hipparcos and Tycho Catalogues, ESA SP-1200

Fenner, Y., Prochaska, J. X., \& Gibson, B. K. 2004, ApJ, 606, 116

Fuhrmann, K. 1998, A\&A, 338, 161

Fuhrmann, K. 2000, http://www . xray.mpe.mpg.de/ fuhrmann

Goswami, A., \& Prantzos, N. 2000, A\&A, 359, 191

Gratton, R. G., Carretta, E., Desidera, S., et al. 2003, A\&A, 406, 131

Grevesse, N., \& Sauval, A. J. 1998, Space Sc. Rev., 85, 161

Hoffman, R. D., Woosley, S. E., Fuller, G. M., \& Meyer, B. S. 1996, ApJ, 460, 478

Holweger, H., \& Müller, E. A. 1974, Sol. Phys., 39, 19

Høg, E., Fabricius, C., Makarov, V. V., et al. 2000, A\&A, 355, L27

Iwamoto, K., Brachwitz, F., Nomoto, K., et al. 1999, ApJS, 125, 439

Kurucz, R. L., Furenlid, I., Brault, J., \& Testerman, L. 1984, Solar Flux Atlas from 296 to 1300 nm, National Solar Observatory, Sunspot, New Mexico
Langer, N., Arcoragi, J.-P., \& Arnould, M. 1989, A\&A, 210, 187

Mashonkina, L., Gehren, T., Travaglio, C., \& Borkova, T. 2003, A\&A, 397, 275

Matteucci, F., Raiteri, C. M., Busso, M., Gallino, R., \& Gratton, R. 1993, A\&A, 272, 421

Mitchell, W. E., \& Mohler, O. C. 1965, ApJ, 141, 1126

Mishenina, T. V., Kovtyukh, V. V., Soubiran, C., Travaglio, C., \& Busso, M. 2002, A\&A, 396, 189

Nissen, P. E. 2004, in Origin and Evolution of the Elements, ed. A. McWilliam, \& M. Rauch (Cambridge: Cambridge Univ. Press), Carnegie Observatories Astrophys. Ser., 4, in press

Nissen, P. E., Chen Y. Q., Asplund, M., \& Pettini, M. 2004, A\&A, 415, 993

Nissen, P. E., \& Schuster, W. J. 1997, A\&A, 326, 751

Olsen, E. H. 1983, A\&AS, 54, 55

Olsen, E. H. 1993, A\&AS, 102, 89

Olsen, E. H. 1994, A\&AS, 106, 257

Pettini, M., Ellison, S. L., Steidel, C. C., \& Bowen, D. V. 1999, ApJ, 510,576

Primas, F., Reimers, D., Wisotzki, et al. 2000, in The First Stars, ed. A. Weiss, T. Abel, \& V. Hill (Berlin: Springer), 51

Prochaska, J. X., Naumov, S. O., Carney, B. W., McWilliam, A., \& Wolfe, A. M. 2000, AJ, 120, 2513

Prochaska, J. X. 2003, in Origin and Evolution of the Elements, ed. A. McWilliam, \& M. Rauch (Cambridge: Cambridge Univ. Press), Carnegie Observatories Astrophys. Ser., 4, in press

Prugniel, P., \& Soubiran, C. 2001, A\&A, 369, 1048

Reddy, B. E., Tomkin, J., Lambert, D. L., \& Allende Prieto, C. 2003, MNRAS, 340, 304

Shetrone, M., Venn, K. A., Tolstoy, E., et al. 2003, AJ, 125, 684

Schuster, W. J., \& Nissen, P. E. 1988, A\&AS, 73, 225

Schuster, W. J., Parrao, L., \& Contreras Martínez, M. E. 1993, A\&AS, 97, 951

Sneden, C., Gratton, R. G., \& Crocker, D. A. 1991, A\&A, 246, 354

Thévenin, F., \& Idiart, T. 1999, ApJ, 521, 753

Thielemann, F.-K., Hashimoto, M., \& Nomoto, K. 1990, ApJ, 349, 222

Timmes, F. X., Woosley, S. E., \& Weaver, T. A. 1995, ApJS, 98, 617

Umeda, H., \& Nomoto, K. 2002, ApJ, 565, 385

Unsöld, A. 1955, Physik der Sternatmosphären (Berlin: Springer Verlag)

Vladilo, G. 2002, A\&A, 391, 407

Woosley, S. E., \& Weaver, T. A. 1995, ApJS, 101, 181

Woosley, S. E., Wilson, J. R., Mathews, G. J., et al. 1994, ApJ, 433, 229 